\title{
Bilateral macular dysplasia ('colobomata') and congenital retinal dystrophy
}

\author{
A T MOORE, D S TAYLOR, AND ANN HARDEN \\ From the Hospital for Sick Children, Great Ormond Street, London WC1N3JH
}

SUMmaRY Three unrelated patients with bilateral macular dysplasia ('colobomata') with no relevant family history were found to have absent or substantially abnormal electroretinograms, implying that there was an associated retinal dystrophy. This may suggest that the macular lesions are associated with a global failure of retinal development, with a regional preponderance rather than a purely localised cause such as an intrauterine infection. It is important to distinguish between congenital infections such as toxoplasmosis and developmental macular colobomata, which have a somewhat similar ophthalmoscopic appearance as a cause of bilateral macular abnormalities seen in young children, since they have different implications for genetic advice and future ophthalmic care.

Until intrauterine infection with Toxoplasma gondii was recognised as a cause of certain congenital macular abnormalities and serological tests for toxoplasmosis became available, many macular lesions which are now more readily diagnosed as those of congenital toxoplasmosis were classified as congenital macular 'colobomata.' Ida Mann pointed out that not only were most of these cases due to intrauterine infection, but the term macular 'coloboma' is itself a misnomer, as it does not result from a defect in closure of the fetal fissure.' The term macular dysplasia may be preferable. There is, however, a group of patients with bilateral macular 'colobomata' or dysplasia, in whom the defect is thought to result from an abnormality of development of the macula unrelated to fetal fissure closure defects. A developmental mechanism is likely in those cases with a family history, or when there are other systemic abnormalities which form part of a recognisable syndrome. It may not be possible to distinguish between inflammatory and developmental aetiology on clinical appearance alone. ${ }^{2}$

Bilateral macular 'colobomata' have been described in sibs $^{36}$ and in two or more successive generations, ${ }^{27-10}$ and have been reported in association with two different systemic abnormalities. Such an association has been proposed as evidence of primary developmental aetiology for the macular Correspondence to Mr D S Taylor, FRCS. lesions. Sorsby ${ }^{2}$ described a family group consisting of a mother and five children all of whom had bilateral pigmented 'colobomata' in association with a rare skeletal abnormality - apical dystrophy of the hands and feet. Bilateral macular 'colobomata' have also been described in idiopathic infantile hypercalcuria." "12 This condition may be inherited in an autosomal recessive manner, as it has been reported in two sibs from a consanguineous marriage."

A third group of patients with macular 'colobomata' have electrophysiological evidence of a more widespread retinal dystrophy. An absent or abnormal electroretinogram has been reported in association with familial macular 'colobomata' ${ }^{39}$ and in 'colobomata' associated with idiopathic infantile hypercalcuria." Macular 'colobomata' and an absent electroretinogram have also been reported in Leber's amaurosis ${ }^{1314}$ and retinitis pigmentosa. ${ }^{15}$ Not all familial cases have abnormal electrodiagnostic tests, however. Tamaki et al. ${ }^{10}$ found a normal electroretinogram, electroculogram, and dark adaptometry in all affected family members in their pedigree of autosomal dominantly inherited macular 'colobomata.'

We present here three cases of bilateral macular dysplasia with abnormal electrodiagnostic findings, in whom the macular lesions are likely to be developmental in origin rather than secondary to intrauterine infection. 


\section{Case reports}

CASE 1

This female infant was first seen at the Hospital for Sick Children at 13 months $^{s}$ of age. She was the second child born to unrelated parents, and there was no relevant family history. The mother had two upper respiratory tract infections during pregnancy at three and eight months, but the pregnancy was otherwise uneventful. There was a normal delivery at term. At birth the infant was noted to have an extra thumb on the right hand (Fig. 1), but initial examination was otherwise normal. The extra digit was excised at 11 months. At 4 weeks the parents had noticed that the child had 'wobbly eyes' and that her vision appeared very poor. She was seen by a local ophthalmologist, who found poor visual attention, nystagmus, and bilateral macular abnormalities.

When the child was first examined at the Hospital for Sick Children she showed no response to light; there were 'roving eye movements' and absent pupillary responses to light. The anterior segments were normal, but both fundi showed similar abnormal appearances (Fig. 2). Both discs were pale, and the retinal blood vessels were attenuated. There were scattered peripheral retinal pigmentation and bilateral non-pigmented macular 'colobomata.' In the right eye there was an excavated, non-pigmented 'coloboma' with ectatic sclera at the base. A few large choroidal blood vessels were visible at the base, but no retinal vessels crossed the defect. In the left eye

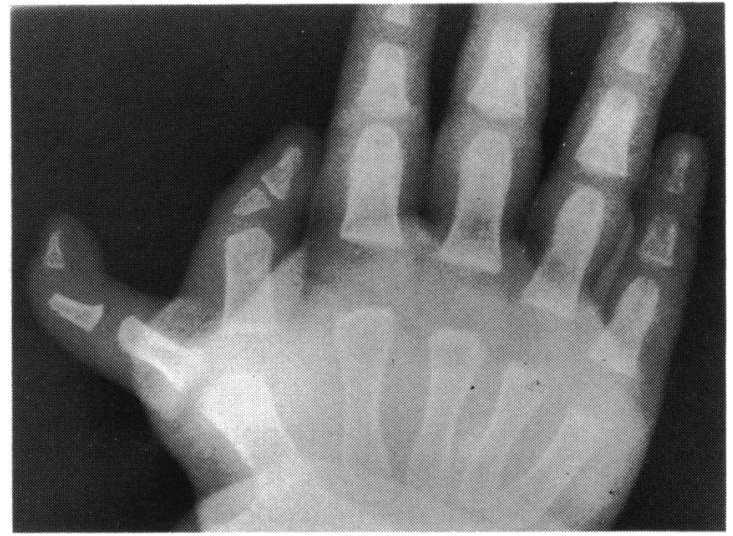

Fig. 1 Case 1. Plain x-ray of right hand showing polydactylism.

there was a non-ectatic macular 'coloboma' with small and medium sized choroidal vessels visible in the base. Again no retinal vessels crossed the defect. General examination was normal apart from a scar at the right thumb. Developmental assessment revealed that she was developing normally for a blind child of her age. There was no recordable electroretinogram or flash visually evoked potential on two occasions (Fig. 3). $X$-ray of the hands and feet showed a short right first metacarpal with an abnormal epiphysis of the terminal phalynx, but was otherwise normal. A CT scan showed high density areas in the retina of both globes, which may represent calcification in the

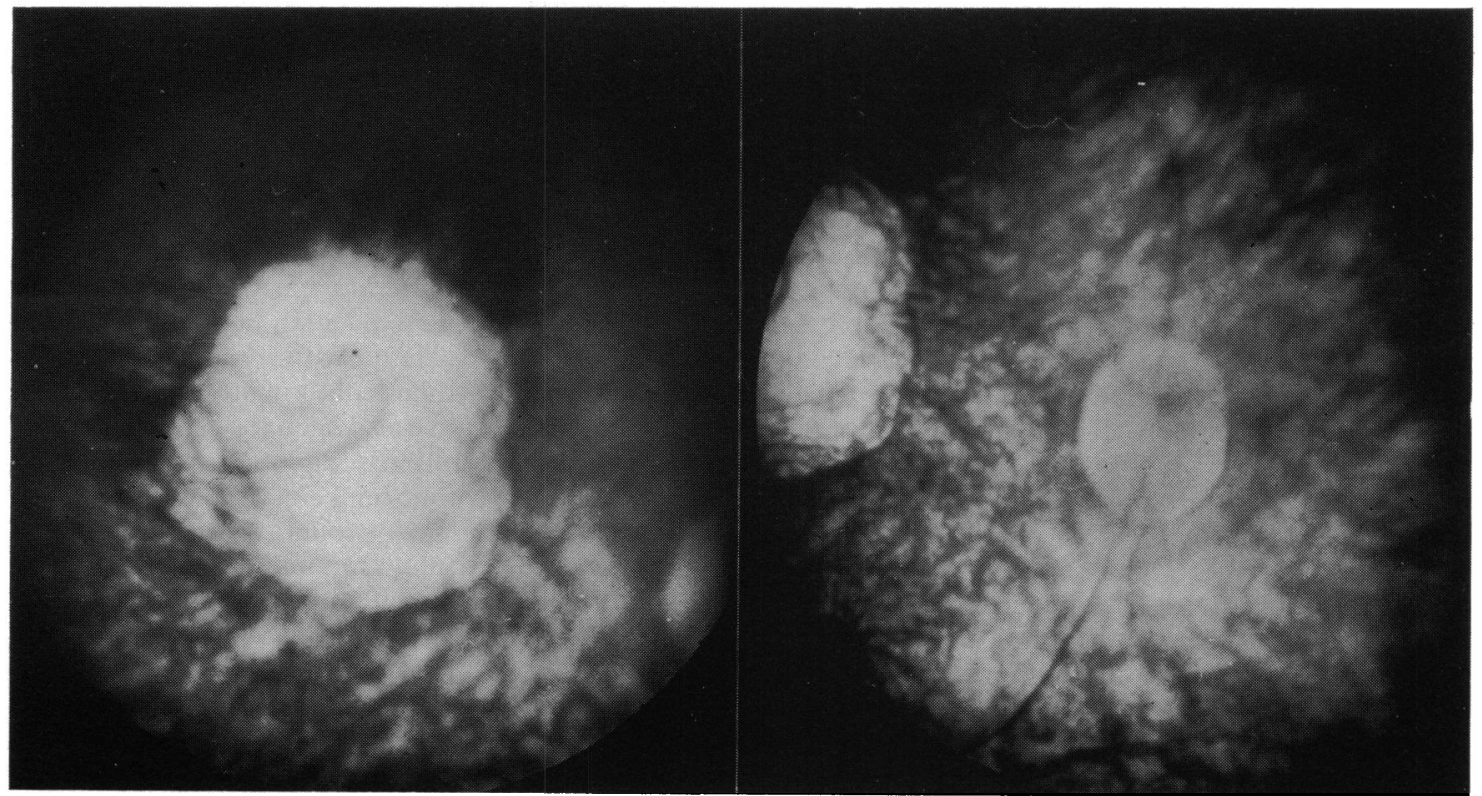

Fig. 2a Case 1. Fundus photograph of right eye showing pole disc with thready vessels and a non-pigmented ectatic macular coloboma, with a few choroidal vessels in the base. 


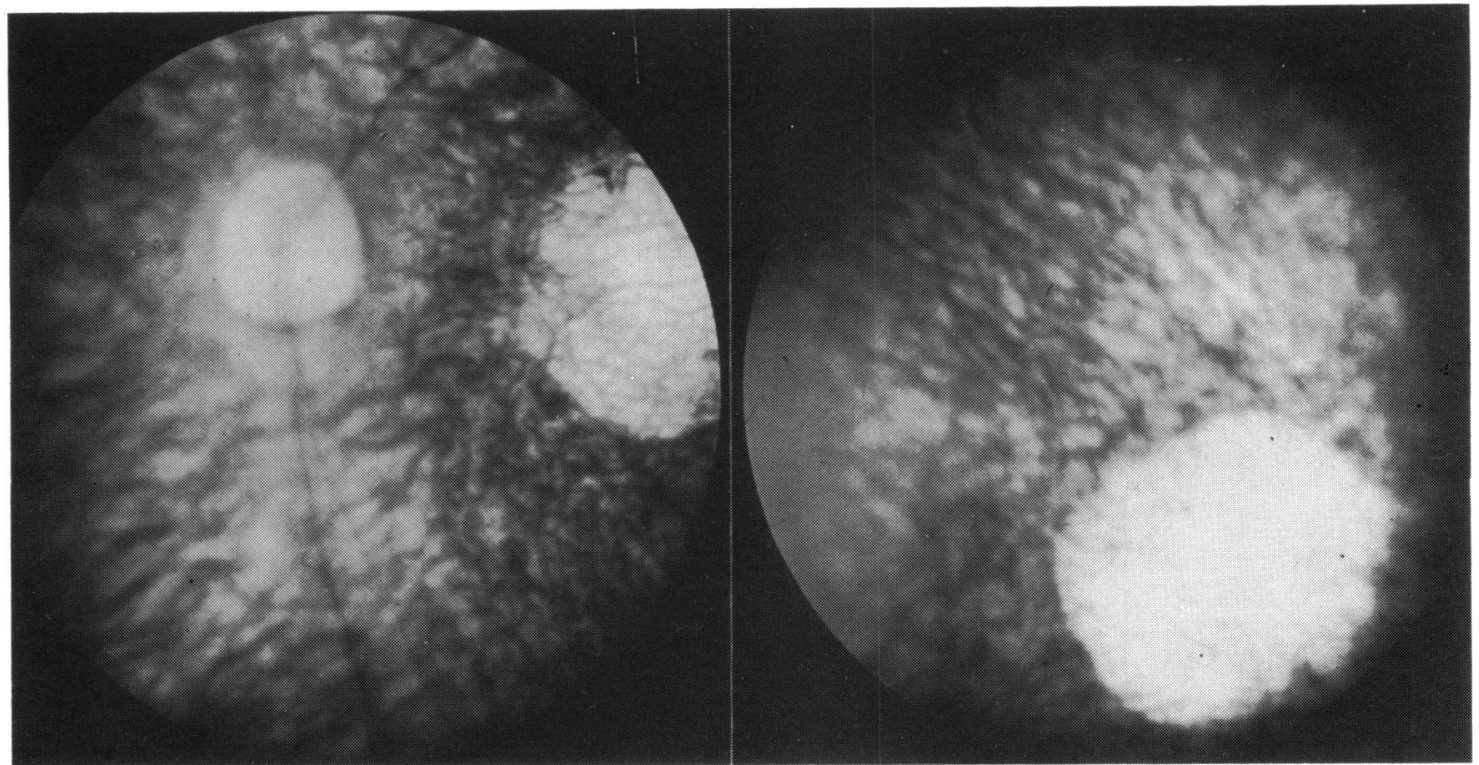

Fig. 2b Case 1. The left eye was similar to the right, but the coloboma was non-ectatic.

base of the macular 'coloboma' (Fig. 4), but was otherwise normal. Other investigations including serum and urinary amino acids, urinary reducing substances, urinary calcium excretion, synthetic vasopressin urinary concentration tests, and skull $x$-ray were normal. Toxoplasma dye test was negative at 1 in 16 dilution in the mother and baby on two occasions. Serological tests for cytomegalovirus, rubella, and herpes simplex were negative in mother and child. The fundi of both parents and her older sibling were examined and found to be normal.

\section{CASE 2}

This young girl was first seen at the Hospital for Sick Children at 3 years of age. She is the only child born to unrelated parents and there is no relevant family history. The mother had severe essential hypertension and depression, and was taking methyldopa, propranolol, and amitriptyline during the pregnancy. She required several admissions during the pregnancy, because of the raised blood pressure, and the delivery was at 38 weeks by caesarian section. Birth weight was $2040 \mathrm{~g}$. The placenta was small and there was slight meconium staining at delivery. At 6 weeks her mother had noticed 'wobbly' eyes and thought that her vision was poor. However, over the following months her vision appeared to improve. When the girl was first seen at the age of 3 years there was horizontal nystagmus, with an abnormal head posture and a face turn to the left. There was a right divergent squint, but ocular movements were full. Both fundi were abnormal (Fig. 5). The discs were normal, but the vessels, which were of normal calibre, were dragged temporally to large, deep, nonpigmented macular defects. There was marked bilateral scleral ectasia with a line of pigment and blood vessels stretched across the defect, giving it a bilobed appearance. Large choroidal vessels crossed the base of the 'coloboma' in each eye. General examination had revealed no other systemic abnormalities. She has shown normal intellectual development; though she has been investigated on several occasions for poor weight gain and short stature, no organic cause has been found. A toxoplasma dye test carried out at the age of 6 months was negative at 1 in 16 dilution.

She was last reviewed in May 1983 at the age of 6, when she was managing well at a normal school. Distance visual acuity was $6 / 36$ in each eye and binocular near vision $6 / 9$ on a reduced Snellen chart at $10 \mathrm{~cm}$. There was horizontal nystagmus, an abnormal head posture, with either left or right face turn, and an alternating divergent squint. Colour vision was normal on testing with Ishihara plates in each eye. Visual field examination showed bilateral relative central scotoma with normal peripheral fields. The fundus appearances were unchanged. The electroretinogram was of markedly reduced amplitude (Fig. 3). The flash visually evoked potential was of normal latency and wave form, but the major early component was of reduced amplitude.

\section{CASE 3}

This Greek boy was first seen at the Hospital for Sick 


\section{ERG}

\section{VEP}
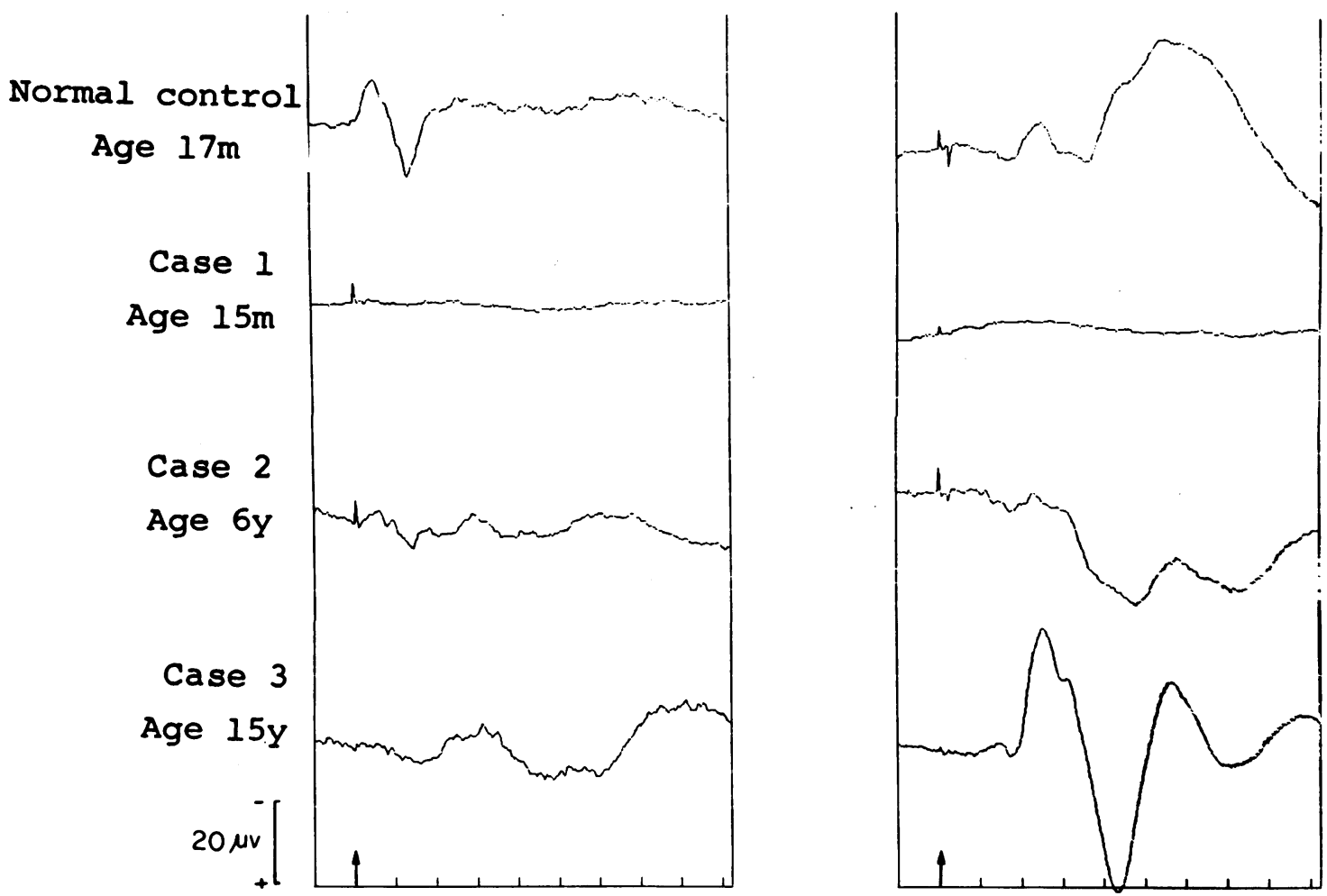

Time marker $25 \mathrm{~ms}$

Fig. 3 ERG (recorded from bridge of nose) and VEP (midoccipital) in response to flash stimulus. Eyes open; both eyes stimulated simultaneously at arrow. Note absent or very poor ERG in cases 1,2, and 3 compared with normal control. In case 1 no VER is recordable; in case 2 the VEP earlier components in the first 75 ms are small and poorly defined compared with normal. In case 3, however, a well defined VEP is seen which is somewhat unusually large, but within normal limits. These findings contrast with cases of toxoplasmosis retinitis which have not shown gross ERG abnormalities.

Children at the age of 15 years. He had been referred to Professor Otto Wolff for investigation of renal, metabolic, and respiratory problems. He was born to unrelated parents at 28 weeks gestation. The pregnancy had been normal until that point. Birth weight was $1500 \mathrm{~g}$, and he required oxygen, without mechanical ventilation, for about 30 days. He had poor vision and nystagmus from infancy but was able to attend a normal school, where he was said to be 'slow.' He also had hearing problems and had worn a hearing aid from the age of 6 . At 10 years he had been noted to get short of breath very easily, and his respiratory difficulties gradually increased, which led to investigation in Greece. This had revealed polycythaemia, interstitial lung disease, and radiological and biochemical evidence of rickets.

When he was examined in London his visual acuity was $3 / 60$ right and $6 / 60$ left, with $+5 \cdot 00$ spherical dioptre correction in each eye. Binocular visual acuity was $6 / 60$ for distance and $6 / 18$ Snellen for near. With a low-vision aid he was able to read a $6 / 6$ reduced Snellen chart at $10 \mathrm{~cm}$. There was manifest horizontal nystagmus and an alternating divergent squint. Ocular movements were full and pupil reactions were normal. Both fundi were abnormal (Fig. 6).

In the right eye there was a deeply excavated macular defect with two large choroidal vessels, one of which appeared to penetrate the sclera in the manner similar to a vortex vein, crossing the base. A thin membrane containing pigment was suspended across the defect at the level of the normal retina. The optic disc was vertically oval and was segmentally hypoplastic, being deficient in the area of the papillo- 


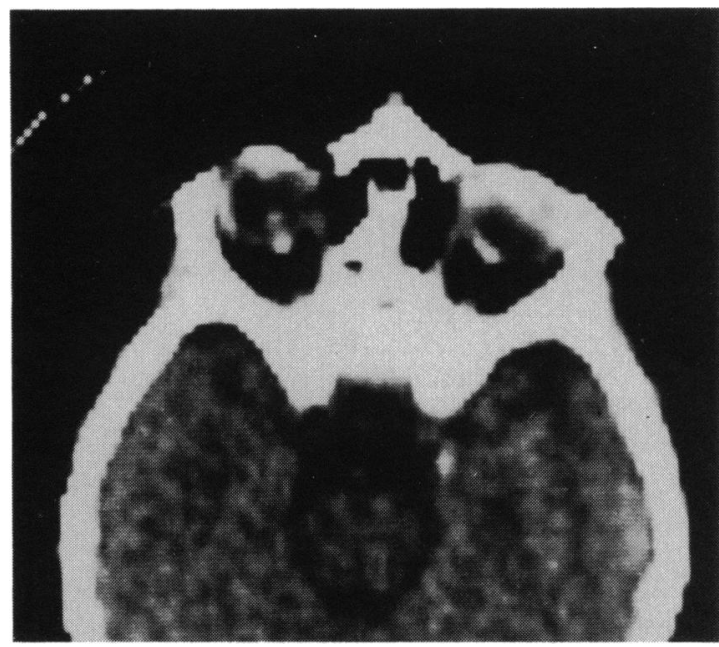

Fig. 4 Case 1. CT scan (EMI 1010) showing high density areas at both posterior poles.

macular bundle-resulting in a kidney-shaped disc. In the left eye the optic disc was similar in shape and there was a large macular defect, but no underlying scleral ectasia. The retinal pigment epithelium and small choroidal vessels were absent in the area of the defect, but medium and large sized choroidal vessels traversed the base. A few retinal vessels crossed the periphery of the lesion. The peripheral retina was normal in each eye. The electroretinogram was reduced in amplitude (Fig. 3). The flash visually evoked potential was well formed and of the usual latency and waveform.

General examination showed that he was below the tenth percentile for both height and weight, and the head circumference was on the third percentile. He was slightly cyanosed, with finger clubbing, but chest examination was normal. He was of subnormal intelligence, had marked genu valgum, but systemic examination was otherwise normal. Extensive investigations were carried out to elucidate the cause of his systemic and ocular problems and to try to provide a single unifying diagnosis. This was not possible. A chest $x$-ray showed the appearance of interstitial lung disease, and lung function tests confirmed that there was a diffusion defect. A lung biopsy, however, was not diagnostic; the histological appearances were consistent either with a viral pneumonia or a disease characterised by circulating immune complexes. Renal investigation revealed renal tubular abnormalities with renal tubular proteinuria, aminoaciduria, glycosuria, and an inability to acidify or concentrate the urine normally. Renal ultrasound and intravenous pyelogram were normal. A renal biopsy showed mild mesangial proliferation and tubular atrophy. There was no interstitial nephritis, and immunofluorescence tests were negative for IgG, IgA, and IgM. A skeletal survey showed radiological evidence of healing rickets. A CT scan showed colpocephaly (Fig. 7). An audiogram revealed bilateral sensorineural hearing loss. A toxoplasma dye test was negative at 1 in 16 dilution, and venereal disease reference laboratory (VDRL) and Treponema pallidum haemagglutination (TPHA) tests were negative. Many other investigations including immunological studies and viral serology were negative. No unifying diagnosis was made. He was treated empirically with oral steroids with some improvement in his respiratory function.

\section{Discussion}

Ida Mann' ${ }^{17}$ classified macular 'colobomata' into three types based on their ophthalmoscopic appearance. The first and commonest type, the pigmented macular 'coloboma,' consists of a circular patch at the macula covered by an irregular mass of pigment, beneath which the choriocapillaris is absent, but small patches of bare sclera and a few choroidal vessels may be seen. There is no scleral ectasia, and normal retinal arterioles pass over the pigmented area, indicating that the main lesion is deep to the inner retinal layers at least. The second type, the nonpigmented 'coloboma,' Mann describes as a round or oval patch at the macula with a white base of bare sclera which is usually ectatic to some extent. There is often pigment clumping at the edge of the lesion, and retinal vessels stop at the margin of the coloboma, suggesting that in this type the retina is also involved. A third, rare, type is represented by those cases in which the macular 'coloboma' is associated with abnormal vessels which may form an anastomosis within the 'coloboma' or run forward from the lesion into the vitreous. ${ }^{1819}$ Not all cases of macular 'colobomata' fall clearly into one of these three groups, and intermediate types do occur. Each of our three cases had macular lesions of the non-pigmented type, and they also do not represent a completely homogeneous group. Mann has speculated that the three types of lesions are caused by a destructive process affecting the developing macular region occurring during the eighth or ninth month, fifth or sixth month, and second or third month of gestation respectively.

The various theories put forward to account for the production of these macular lesions have been reviewed by Mann ${ }^{117}$ and Sorsby. ${ }^{2}$ They fall into two main groups. Firstly, there may be a failure of normal development of the eye, giving rise to the macular abnormality; or, secondly, the development of the eye may be initially normal but later become disorganised by a pathological process, such as infection 

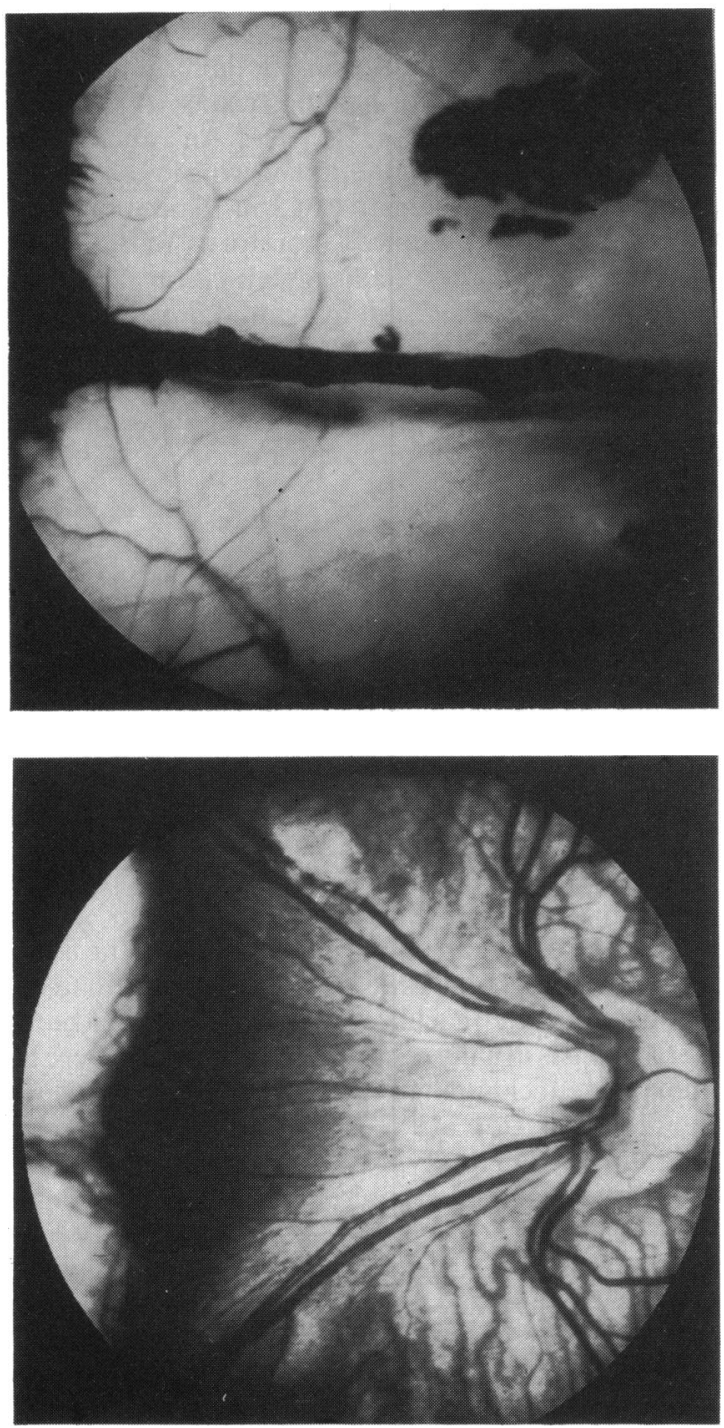

in intrauterine life or soon after birth. Although there is no satisfactory explanation for the production of macular 'colobomata' by a failure of development which takes into account both the normal embryological development of the eye and the clinical features of the macular lesions, ${ }^{217}$ the presence of scleral ectasia and in case 3, the hypoplastic discs may indicate that the causative lesion occurs very early in development.

Most cases with the typical ophthalmoscopic appearance are undoubtedly due to intrauterine infection with Toxoplasma gondii. It is also possible that other infective agents may produce similar macular lesions, but this mechanism will not account for the occurrence of macular 'colobomata' in

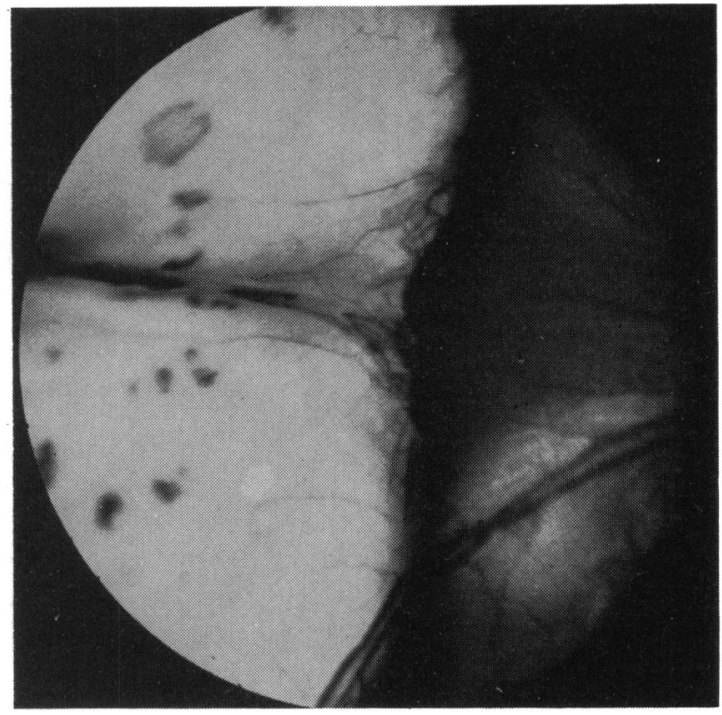

Figs. 5a and 5b Case 2. Both fundi showing the optic disc vessels being dragged temporally towards huge excavated colobomata, which were divided into two 'lobes' by a horizontal band of vessels and pigment.

families, and in association with other specific congenital abnormalities. Congenital toxoplasmic chorioretinitis has never been confirmed in siblings and results only from infection of a non-immune mother in pregnancy. ${ }^{20}$ Other hereditary macular conditions, such as central areolar pigment epithelial dystrophy ${ }^{2122}$ and progressive bifocal chorioretinal atrophy, ${ }^{23}$ may have a similar ophthalmoscopic appearance, but the clinical progression of these conditions in childhood and the absence of scleral ectasia serve to differentiate them from true macular 'colobomata.'

Each of our three patients had a toxoplasma dye test which was negative at 1 in 16 dilution, which makes systemic toxoplasmosis unlikely, since the 


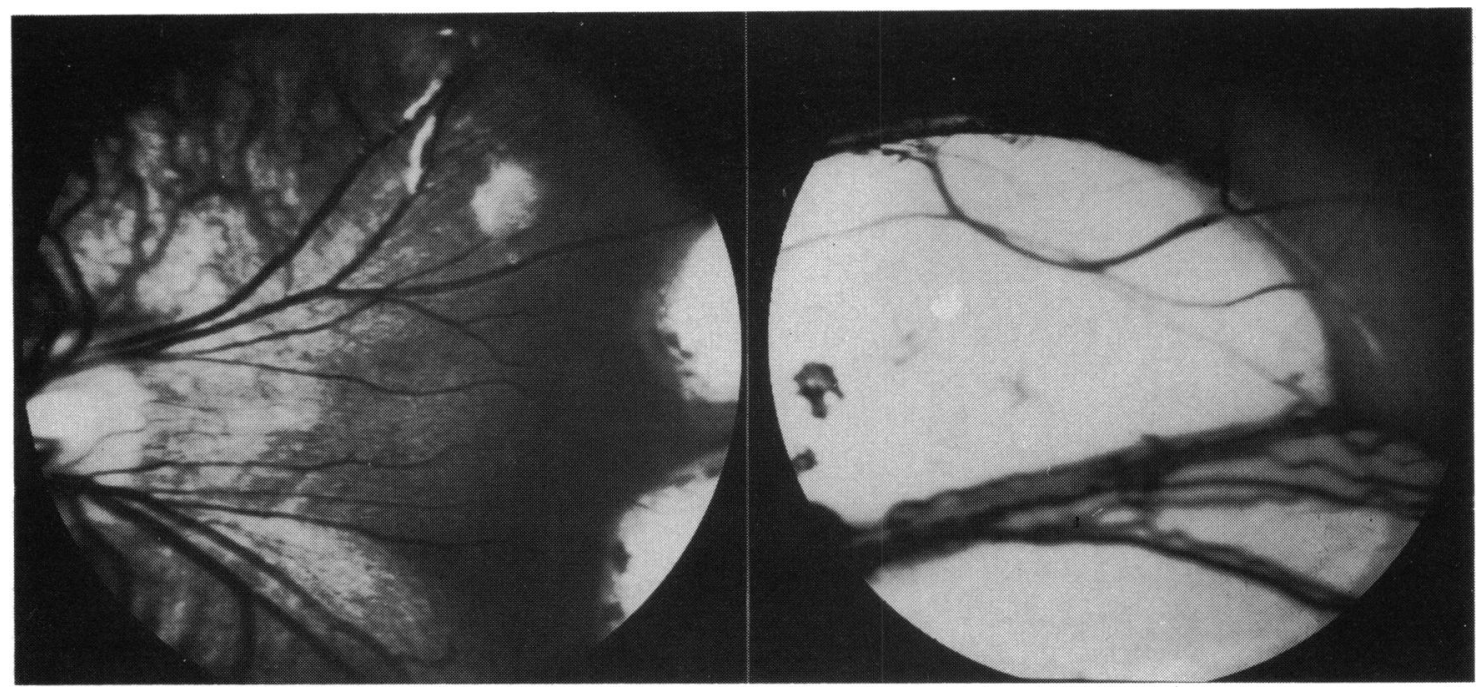

Fig. 5b Legend under Fig. 5a.

toxoplasma titre tends to parallel systemic involvement. The serological results do not, however, exclude congenital toxoplasmosis confined to the eye. Zscheile ${ }^{24}$ has reported a case of ocular toxoplasmosis confirmed by histopathology in whom the toxoplasma dye test was positive only in undiluted serum. Any toxoplasma titre may be significant in the presence of typical chorioretinal lesions. ${ }^{25}$ However, each case reported here had an absent or substan- tially abnormal electroretinogram, which cannot be explained on the basis of a single macular toxoplasma lesion. The fact that the electroretinogram is abnormal in familial macular 'colobomata' and those occurring as part of a recognisable syndrome points to a developmental origin for the macular lesions in our patients.

Macular dysplasia has been reported in association with several different skeletal abnormalities. ${ }^{2426}$

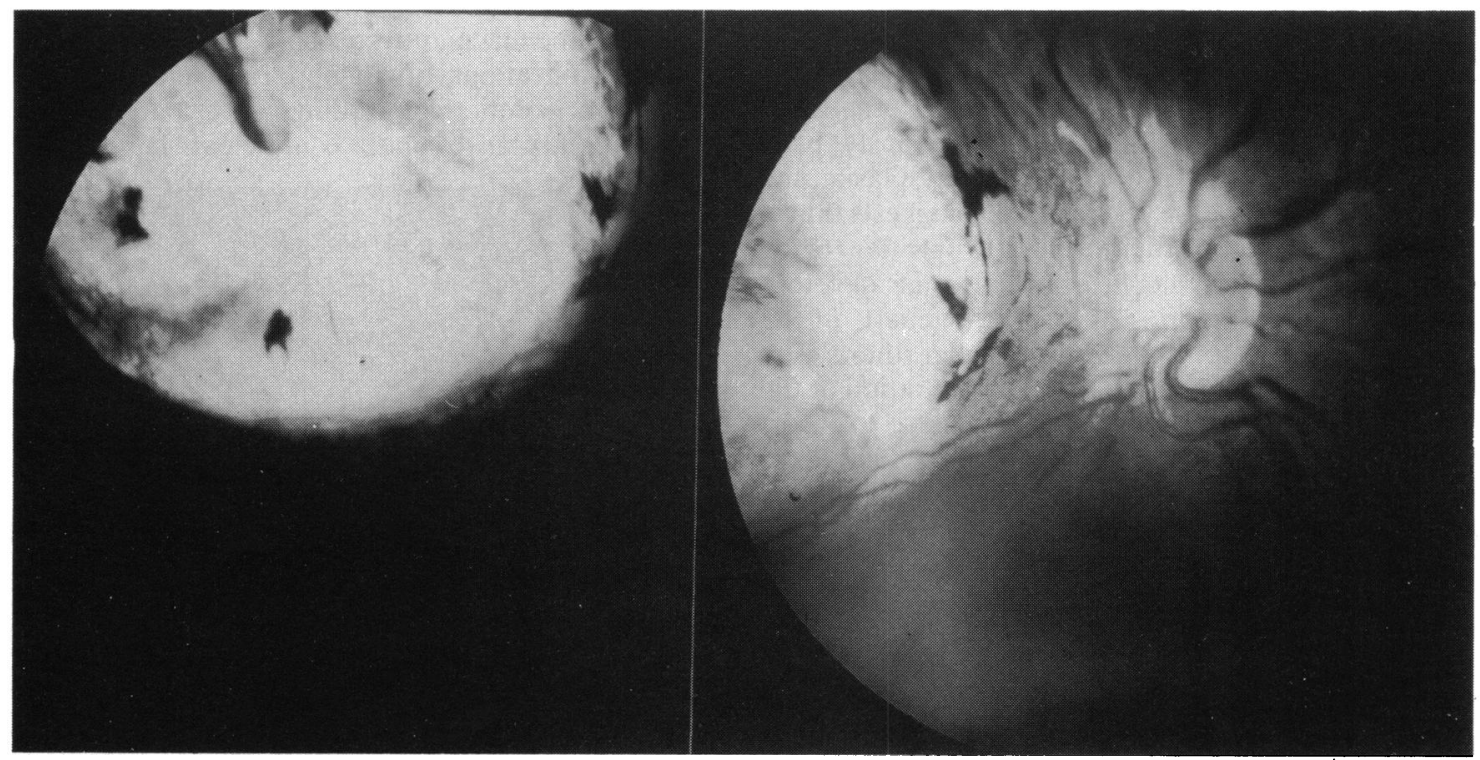

Fig. 6a Case 3. The right fundus shows a hypoplastic optic disc with the temporal segment being most affected. The macular area is replaced by a circumscribed ectatic area at the base of which was a large choroidal vessel. A thin membrane containing a few spots of pigment covered the coloboma at the level of the retina. 


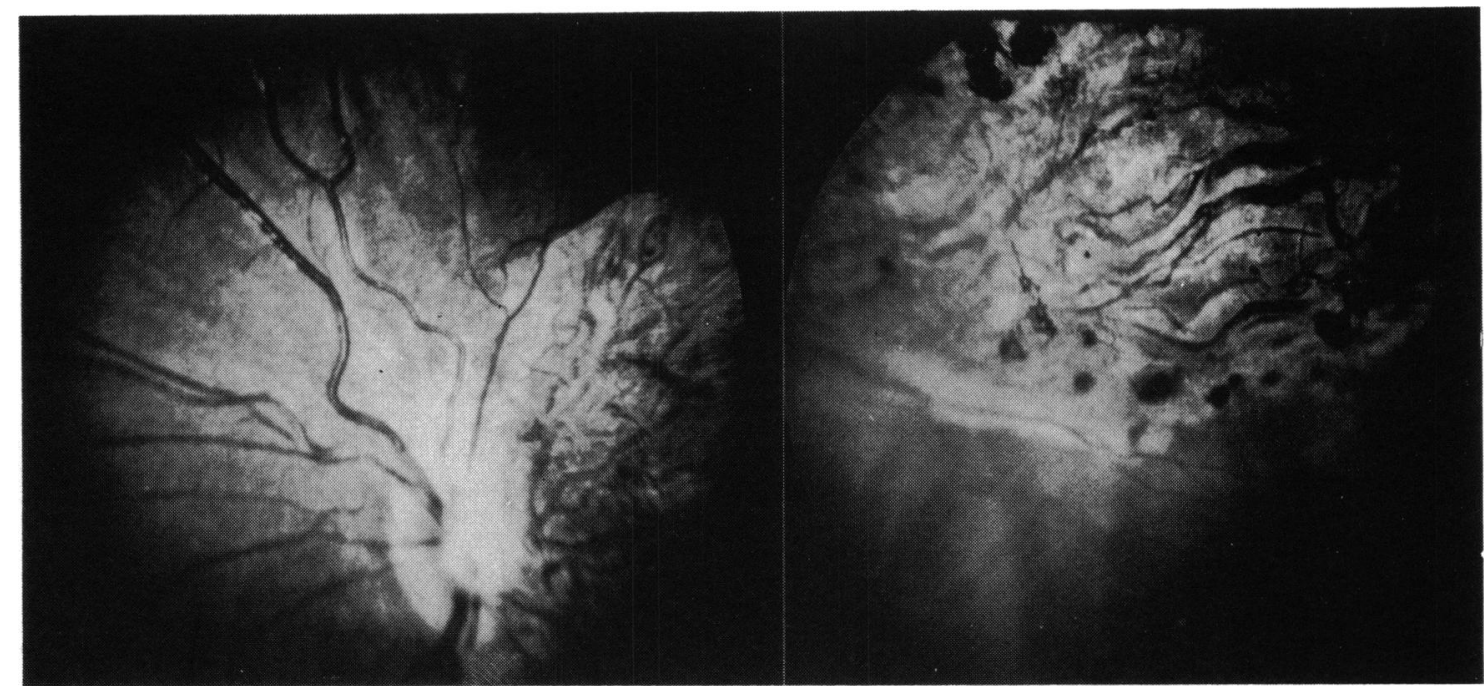

Fig. 6b Case 3. The left fundus was similar but showed better preserved choroid vessels and pigment, and there was no scleral ectasia.

Case 1 had skeletal abnormalities consisting of an extra digit but showed none of the other features of apical dystrophy and macular 'colobomata' described by Sorsby. ${ }^{2}$ It is interesting that our patient had an extra thumb, as the most constant radiological feature in Sorsby's family was a bifid terminal phalynx of the thumbs and great toes.

The third case reported here had several systemic problems which could not be related to his ocular disease. In addition to the macular lesions both discs were hypoplastic (Fig. 6), the nerve head being especially deficient on the temporal side where the papillomacular bundle normally enters the disc. Optic nerve hypoplasia has not previously been reported in association with macular dysplasia, and the presence of the disc abnormalities suggests that in this case the insult to the developing macular region occurred at an early stage in intrauterine development. The early theories of the pathogenesis of the optic nerve hypoplasia proposed that there is a primary failure of ganglion cell differentiation at the 13-15 mm stage (6 weeks) or their failure to incorporate into the optic stalk at the $19 \mathrm{~mm}$ stage (7 weeks). ${ }^{27}$ However, the frequent association of optic nerve hypoplasia with central nervous system (CNS) malformations, in particular midline abnormalities such as absent or anomalous septum pellucidum $^{2829}$ or abnormalities of the corpus callosum or with cerebral atrophy ${ }^{31}$ and porencephaly, ${ }^{31}$ has led to the suggestion that optic nerve hypoplasia may also result from secondary atrophy of the optic nerves during intrauterine development, either as a result of transynaptic degeneration or by a failure of normal cortical induction or influence on optic nerve development. ${ }^{30}$ It is also possible that an insult causing a CNS abnormality may also simultaneously damage the developing optic nerve. The optic nerve hypoplasia in our patient may have been related to his CNS abnormality (Fig. 7); optic nerve hypoplasia has been reported in association with colpocephaly..$^{32}$ However, the unusual configuration of the hypoplastic discs, with the main defect being related to the deficient papillomacular bundle suggests that the optic nerve hypoplasia in this case is related to the macular dysplasia.

The implications of an abnormal electroretinogram for genetic counselling in a patient with bilateral macular dysplasia is not clear. It is evident

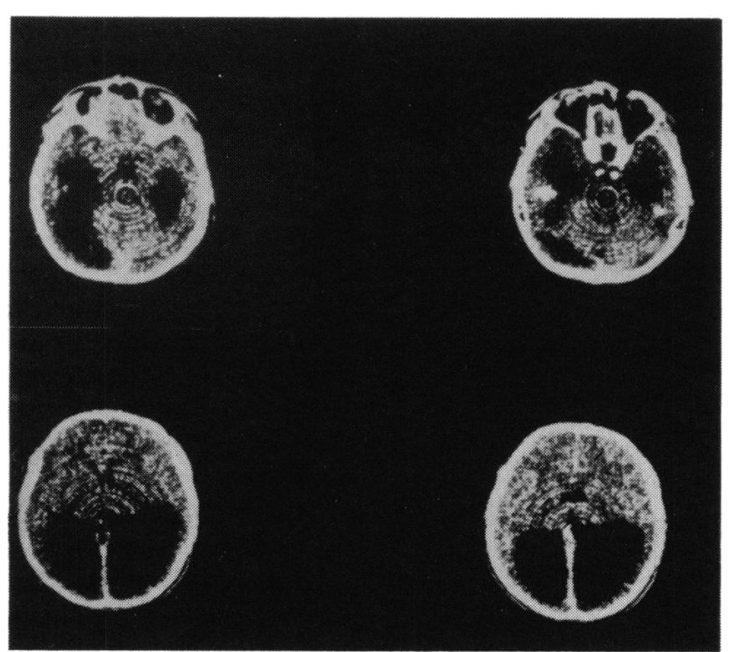

Fig. 7 Case 3. CT scan showing colpocephaly. 
that patients with bilateral macular dysplasia form a heterogeneous group; macular 'colobomata' (dysplasia) have been reported to have both dominant and recessive modes of inheritance. Dominantly inherited macular 'colobomata' may occur alone ${ }^{910}$ or with a specific skeletal abnormality ${ }^{2}$; the electroretinogram may be normal ${ }^{10}$ or abnormal. ${ }^{9}$ An autosomal recessive mode of inheritance is seen with macular 'colobomata' in association with retinitis pigmentosa $^{15}$ and Leber's amaurosis ${ }^{1314}$ and is the possible mode of inheritance in 'colobomata' associated with idiopathic infantile hypercalcuria." The two cases reported by Phillips and Stokoe ${ }^{3}$ also occurred in siblings. All these patients had absent or severely abnormal electroretinograms. It is difficult to give accurate genetic advice to parents who have had one child with isolated macular 'colobomata' and no family history, especially since the condition may have different aetiologies. If the child has an absent electroretinogram and has had poor vision and nystagmus from infancy, the condition is more likely to resemble Leber's amaurosis in being inherited as an autosomal recessive condition.

Our three cases also illustrate the variability of visual acuity in this condition. Case 1 behaved as a blind child, whereas the other two patients achieved useful vision despite extensive macular lesions and an absent or abnormal electroretinogram. Therefore when a child is seen in infancy with bilateral macular dysplasia it may be better to give the parents a cautiously optimistic visual prognosis, as the vision may later prove to be better than expected from the ophthalmoscopic appearance, though the long term course of the retinal dystrophy is less certain. Indeed Clarke $^{33}$ in 1927 described a child with bilateral macular 'colobomata' to whom he gave a 'gloomy' visual prognosis who later went normally through Harrow School, took a degree at Cambridge, and was always good at games, especially tennis. There may be several interpretations of this achievement.

We thank Professor $\mathrm{O} \mathrm{H}$ Wolff for permission to report details of case 3 and Martin Johns for taking the fundus photographs. Mr A T Moore received financial support from the TFC Frost Charitable Trust.

\section{References}

1 Mann IC. On certain abnormal conditions of the macular region usually classified as colobomata. Br J Ophthalmol 1927; 11: 99-116.

2 Sorsby A. Congenital coloboma of the macula together with an account of the familial occurrence of bilateral macular colobomata in association with apical dystrophy of the hands and feet. Br J Ophthalmol 1935; 19: 65-90.

3 Phillips CI, Stokoe NL, Newton M. Macula coloboma and retinal aplasia. Acta Ophthalmol (Kbh) 1981; 59: 894-900.

4 Phillips CI, Griffiths LD. Macula coloboma and skeletal abnormality. Br J Ophthalmol, 1969; 53: 346-9.

5 Schott K. Über das songenannte Kolobom der Makula. Klin Monastsbl Augenheilkd 1921; 67: 415-24.
6 Evans PJ. Familial macular colobomata. Br J Ophthalmol 1937; 21: $503-6$.

7 Clausen M. Typisches beiderseitiges hereditares MakulaKolobom. Klin Monatsbl Augenheilkd 1921; 67: 116.

8 Davenport RC. Bilateral 'macular coloboma' in mother and son. Proc $R$ Soc Med 1927; 21: 109-10.

9 Miller SA, Bresnik G. Familial bilateral macular coloboma. Br J Ophthalmol 1978; 62: 261-4.

10 Tamaki S, Garcia CA, Katzmann J. Macular coloboma. Invest Ophthalmol Vis Sci (Arvo suppl) 1979; abstr 15, 222.

11 Meier W, Blumberg A, Imahorn W, De Luca F, Wildberger H, Oetliker $O$. Idiopathic hypercalcuria with bilateral macular colobomata: a new variant of oculo-renal syndrome. Helv Paediatr Acta 1979; 34: 257-69.

12 Gil-Gilberneau J, Galan A, Callis L, Rodrigo C. Infantile idiopathic hypercalcuria, high congenital myopia and atypical macular coloboma: a new oculo-renal syndrome? J Pediatr Ophthalmol 1982; 19: 7-11.

13 Margolis S, Sher BM, Carr RE. Macular coloboma in Leber's congenital amaurosis. Am J Ophthalmol 1977; 83: 27-31.

14 Leighton D, Harris R. Retinal aplasia in association with macular coloboma, keratoconus and cataract. Clin Genet 1973; 4: $270-4$.

15 Heckenlively JR, Garcia CA, Maumenee IH, Shahininian J. Two retinitis pigmentosa syndromes. Invest Ophthalmol Vis Sci (Arvo suppl) 1979; abstr 3, 119.

16 Freedman J, Gombos GM. Bilateral macular coloboma, keratoconus and retinitis pigmentosa. Ann Ophthalmol 1971; 3: 664-6.

17 Mann I. Developmental abnormalities of the eye. 2nd ed. Philadelphia: Lippincott, 1957; 151-70.

18 Beaumont WM. Macular coloboma? Trans Ophthalmol Soc UK 1891; 11: 221.

19 Silcock QA. Vestigial hyaloid artery starting from a macular coloboma. Trans Ophthalmol Soc UK, 1900; 20: 188.

20 Perkins ES. Ocular toxoplasmosis. Br J Ophthalmol 1973; 57: 1-17.

21 Lefler WH, Wadsworth JAC, Sidbury JB. Hereditary macular degenerations and amino-aciduria. Am J Ophthalmol 1971; 71: 224-30.

22 Hermsen VM, Judisch GM. Central aerolar pigment epithelial dystrophy. Ophthalmologica 1984; 189: 69-72.

23 Waheed AA, Wyse CT. Progressive bifocal chorioretinal atrophy. BrJ Ophthalmol 1968; 52: 742-50.

24 Zscheile FP. Recurrent toxoplasmic retinitis with weakly positive methylene blue dye test. Arch Ophthalmol 1964; 71: 645-8.

25 Smoling G, O'Connor GR. Toxoplasma dye test in ocular immunology. Philadelphia: Lea and Febiger, 1981: 83.

26 Smith RD, Fireman RM, Sillence DO, et al. Brief clinical report: congenital macular colobomas and short limbed skeletal dysplasia. Am J Med Genet 1980; 5: 365-71.

27 Anonymous. Editorial: Small optic discs. Br J Ophthalmol 1978; 62: 1-2.

28 De Morsier G. Etudes sur les dysraphies cranioencephaliques 111. Agénésie du septum lucidum avec malformations due tractus optique la dysplasie septo-optique. Schweiz Arch Neurol Neurochir Pschiatr 1956; 77: 267-92.

29 Hoyt WF, Kaplan SL, Grumbach MM, Glaser JS. Septo-optic dysplasia and pituitary dwarfism. Lancet 1970; ii: 893-4.

30 Rogers GL, Brown D, Gray I, Bremer D. Bilateral optic nerve hypoplasia associated with cerebral atrophy. J Pediatr Ophthalmol 1981; 18: 18-21.

31 Greenfield PS, Wilcox LM, Weiter JJ, Adelman L. Hypoplasia of the optic nerve in association with porencephaly. $J$ Pediatr Ophthalmol 1980; 17: 75-80.

32 Garg BP. Colpocephaly: an error of morphogenesis? Arch Neurol 1982; 39: 243-6.

33 Clarke E. Coloboma at the macula (both eyes). Br J Ophthalmol 1927; 11: 96-9. 